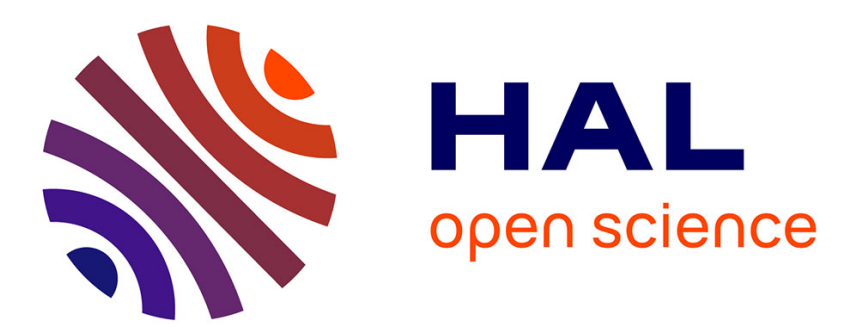

\title{
Experimental characterization of a bi-dimensional array of negative capacitance piezo-patches for vibroacoustic control
}

Flaviano Tateo, Manuel Collet, Morvan Ouisse, Mohamed Ichchou, Kenneth Cunefare, Philippe Abbé

\section{To cite this version:}

Flaviano Tateo, Manuel Collet, Morvan Ouisse, Mohamed Ichchou, Kenneth Cunefare, et al.. Experimental characterization of a bi-dimensional array of negative capacitance piezo-patches for vibroacoustic control. Journal of Intelligent Material Systems and Structures, 2015, 26 (8), pp.952-964. 10.1177/1045389X14536006 . hal-02868325

\section{HAL Id: hal-02868325 \\ https://hal.science/hal-02868325}

Submitted on 15 Jun 2020

HAL is a multi-disciplinary open access archive for the deposit and dissemination of scientific research documents, whether they are published or not. The documents may come from teaching and research institutions in France or abroad, or from public or private research centers.
L'archive ouverte pluridisciplinaire HAL, est destinée au dépôt et à la diffusion de documents scientifiques de niveau recherche, publiés ou non, émanant des établissements d'enseignement et de recherche français ou étrangers, des laboratoires publics ou privés. 
This document is the author's final manuscript of

F. Tateo, M. Collet, M. Ouisse, M.N. Ichchou, K.A. Cunefare \& P. Abbe: Experimental characterization of a bi-dimensional array of negative capacitance piezo-patches for Vibroacoustic Control. Journal of Intelligent Material Systems and Systems, 2014.

This paper has been published by SAGE Publications and can be found at http://dx.doi.org/10.1177/1045389X14536006 


\title{
Contents
}

1 Introduction $\quad 2$

2 System design $\quad 3$

2.1 Plate and periodic array design . . . . . . . . . . . . . . . 4

2.2 Shunt circuit design . . . . . . . . . . . . . . . . . 5

$\begin{array}{llr}3 & \text { Experimental set-up } & 9\end{array}$

4 Results and discussions $\quad 10$

4.1 Single-point mobility measurement . . . . . . . . . . . . . . . . 10

4.2 Third octave mobility comparison . . . . . . . . . . . . . . . . 10

4.3 Full-field mobility measurement . . . . . . . . . . . . . . . . . . . . . . . 12

4.4 Loss factor estimation . . . . . . . . . . . . . . . . . . . 13

4.5 Kinetic energy distribution . . . . . . . . . . . . . . . . . . . . . 15

4.6 Power injected . . . . . . . . . . . . . . . . . 16

$\begin{array}{lll}5 & \text { Conclusions } & 17\end{array}$

\section{Title}

Experimental characterization of a bi-dimensional array of negative capacitance piezopatches for vibroacoustic control

\section{List authors}

F. Tateo, M. Collet, M. Ouisse, M.N. Ichchou, K.A. Cunefare, P. Abbe

\begin{abstract}
This study presents an experimental investigations of the application of a periodic array of shunted piezoelectric patches with negative capacitance for the broadband control of waves propagating on a flexible plate. A $15 \times 5$ array of piezoelectric patches is bonded onto the top surface of a freely-supported rectangular plate. The patch array is intended to serve as an active interface between two regions of the plate, where one region has an input disturbance force and the other does not. Each patch is shunted through a single circuit, reproducing a resistance in series with a negative capacitance. The magnitude of the reactive part of the negative shunting impedance is tuned close to the intrinsic capacitance of the piezoelectric patch. The real part is adjusted for either light damping so as to induce a reactive (reflective) response, or with heavy damping to induce greater absorption. The experimental responses of the system equipped with this active interface display a strong attenuation or reflection of vibrations, depending on the shunt resistance, over a large frequency range, including the mid-frequency regime. In an effort to control vibroacoustic phenomena, this study represents the first attempt to implement an integrated smart metacomposite active interface on a plate structure.
\end{abstract}




\section{Introduction}

The design of innovative structures incorporating multiple physical functionalities represents a big challenge for both industrial and academic communities. Some examples of this new trend can be found in the aerospace sector, where an intensive effort is ongoing to integrate lightweight structures made of composite material. These composite structures bring new fatigue and damage risks, as well as vibration and acoustic problems. Thus, significant effort has been expended to add smart capabilities to integrate structural health monitoring systems and passive or active vibration control devices to composite structures.

This paper is a contribution to the challenges of designing and implementing a new class of integrated smart metacomposites capable of improved engineering performances in terms of mechanical and vibroacoustic behavior as compared to strictly passive structures.

The definition of a metacomposite combines two different aspects of vibration control. The first aspect is connected to periodic structure theories, which are usually associated with metamaterial developments. In the field of light propagation, research has explored how to design and construct photonic crystals exhibiting photonic band gaps that prevent light from propagating in certain directions with specified frequencies. Other efforts have explored creation of photonic crystals able to propagate light in anomalous and useful ways (i.e. negative refraction and artificial magnetism). In the acoustic domain, similar studies were carried out with the aim of preventing the propagation of elastic waves within a medium. For both light and acoustic waves, the band gap is obtained by periodically modulating some electromagnetic or mechanical properties (Yang et al. (2002)). This technique presents two main problems: the spatial modulation must be of the same order as the wavelength in the gap, and the position of the band gap cannot be easily changed since it strongly depends on the materials employed (Bragg's band gap). A possible solution for these problems is found using composites with locally resonant units. The periodicity of the crystal creates a stop band that can be shifted by modifying the properties of the resonators. Liu et al. (2000) had demonstrated that a resonant sonic crystal with building blocks of rubber-coated lead balls exhibits a low-frequency sonic band gap, and the resonance can provide a maximum impedance mismatch to shield against airborne sound. The same effect can be obtained using Helmoltz resonators as showed by Fang et al. (2006), Ambati et al. (2007) and Hu et al. (2005). The same idea was extended in the vibroacoustic domain for the control of elastic waves propagating into a waveguide. The resonant units in this case were obtained using RL circuits shunted to piezoelectric ceramics embedded on the structure's surface. Numerous works have been published that present analyses of the capability and efficiency of a shunted piezoelectric patch for structural damping and wave cancellation (Park and Baz (2005)). An elegant formulation of passive shunting was first proposed by Hagood and von Flotow (1991) and is still commonly used. The study showed how a piezoelectric material shunted through a series RL circuit, i.e., a resonant shunt, which would exhibit a behavior analogous to the well-known mechanical tuned mass damper. Periodically induced impedance-mismatch zones generate broader stop bands, i.e., frequency bands where waves are attenuated. The tunable characteristics of shunted piezopatches allow the equivalent mechanical impedance of the structure to be tuned so that stop bands are generated over desired frequency ranges. The presence of a resistance in the shunt circuit generates a damped resonance of the electrical network. The resistance also allows the energy dissipation mechanism of shunted piezos to be exploited, which dampens the amplitude of vibration also outside the stop bands. The original periodic shunting concept was numerically demonstrated on rods and fluid-loaded axisymmetric shells in 
Thorp et al. (2005). More recently, this strategy was extended to plates in Casadei et al. (2010), Spadoni et al. (2009) and Chen et al. (2013), where the Bloch theorem was used to predict the dispersion properties of the resulting periodic assembly. However the limitation of this approach is the narrow-band effectiveness of resonant circuits. For that reason a different circuit layout was proposed. A very effective method is based on the use of negative capacitance shunts, as originally proposed by Forward (1979). In this configuration, a piezoelectric patch is shunted through a passive circuit to a negative impedance converter. In this way, the internal capacitance of the piezoelectric ceramic is artificially canceled, and the impedance of the shunt circuit reduces to that of the passive circuit. Optimization of the electrical impedance for modal damping is well described in Livet et al. (2011). Although the negative capacitance shunting strategy has been experimentally validated, it must be used with caution since it requires active elements that can destabilize the structure if improperly tuned. This technique requires in fact to tune the circuit very close to the stability limit (Fukada et al. (2004) and Kim and Jung (2006)). The second concept includes the definition of composite conceived in a broader sense, in which shunted piezoelectric materials, electronic components, controllers and the structure are intimately connected to each other. In this respect, the notion of programmable matter coined by Toffoli and Margolus (1991) to refer to an ensemble of computing elements arranged in space is now extended to smart materials based on distributed piezoelectric actuators able to modify the inherent vibroacoustic properties based on an input signal.

In this paper, the metacomposite structure of interest consists of an array of piezoelectric patches periodically arranged over a limited region of the surface of a two-dimensional waveguide. This array of patches is intended to serve as an active interface between regions of the waveguide, formed from a plate. This active interface allows modification of the scattering properties of the waveguide in terms of reflected and absorbed energy. This controlling capability is obtained by correctly tuning the parameters of the external circuit by which almost arbitrary effective structural impedance may be obtained. The selection of the electric component is made according to the methodologies developed by Collet et al. (2012) and subsequently extended to more complicated cases involving fluid-structure interaction in Collet et al. (2012). Experimental validation of this approach has been made on a beam in Beck et al. (2011) and subsequently extended to a flexible plate completely covered by piezoelectric patches in Casadei et al. (2010). The novelty of the paper at hand is to use an array of periodic shunted patches to create a controllable impedance boundary between an "upstream" and "downstream" section of the two-dimensional plate waveguide.

The remainder of this paper is organized into four sections. The first section provides a review of the design of the plate, piezoelectric array, and shunt circuits for the control system applied to the patches in the piezoelectric array. The second section describes the experimental procedures used to investigate the system. The third section presents the experimental results. Finally, the last section summarizes the major findings presented in this paper and provides recommendations for future investigation.

\section{System design}

This section introduces the design of the smart metacomposite, including the plate structure, design criteria leading to selection of the piezoelectric patches used in the active interface, and the design and impedance characteristics of the shunting circuit. 
Table 1: Geometry and physical properties of the system.

\begin{tabular}{llll}
\hline Plate & Length & 2100 & $\mathrm{~mm}$ \\
& Height & 1050 & $\mathrm{~mm}$ \\
Thickness & 3 & $\mathrm{~mm}$ \\
& Mass density & 2700 & $\mathrm{~kg} / \mathrm{m}^{3}$ \\
& Young's modulus & $70 \cdot 10^{9}$ & $\mathrm{~N} / \mathrm{m}^{2}$ \\
& Poisson's ratio & 0.33 & - \\
\hline Piezo & Length & 50 & $\mathrm{~mm}$ \\
& Height & 50 & $\mathrm{~mm}$ \\
Thickness & 0.5 & $\mathrm{~mm}$ \\
& Mass density & 7650 & $\mathrm{~kg} / \mathrm{m}^{3}$ \\
& Poisson's ratio & 0.31 & - \\
Dielectic loss & $<0.05$ & $\%$ \\
Coupling factor & 0.33 & - \\
$\left(k_{31}\right)$ & & \\
\hline
\end{tabular}

\subsection{Plate and periodic array design}

The basic structure of the metacomposite comprises an aluminum plate and array of piezoelectric patches as depicted in Figure 1. The geometry and material properties for the plate and piezoelectric materials are listed in Table 1. The plate is suspended from a rigid frame by metallic wires in order to approximate free-free boundary conditions. The plate is equipped with 75 piezoelectric patches (Ferroperm Industries $P Z 26$ series) arranged in a regular $15 \times 5$ array as seen in Figure 1 and Figure 2. The dimension of the piezoelectric patches are listed in Table 1. The patches and the depth of the patch array were selected based on consideration of their size relative to the shortest structural wavelength of interest. The array was intended to be effective up to a frequency of $5000 \mathrm{~Hz}$. The plate's dispersion relation for the $A_{0}$ flexural mode at $5000 \mathrm{~Hz}$ has a wavelength of $30 \mathrm{~cm}$. With patches having dimensions of $5 \times 5 \mathrm{~cm}$, there will be no charge cancellation on an individual patch as would occur if the patch dimensions were comparable to a wavelength. Further, the depth of the patch array is $35 \mathrm{~cm}$ such that it spans more than a wavelength at 5000 Hz. Since the array elements are uncoupled electronically, each element within the array would still function at the highest frequency of interest, even though the entire array depth would be comparable to a wavelength.

The thickness of the patch was selected considering the constraints of the electric circuit and the nature of the control technique. Different authors have shown that the best controlling effect is obtained when the negative impedance shut circuit is tuned close to the inherent patch capacitance (Forward (1979) and Livet et al. (2011)). This property strongly depends on the material properties and the geometry of the patch. Once the material properties and the two dimensions of the piezoelectric patch are chosen, the only parameter which remains for selection is the thickness. Thin patches exhibit larger values of intrinsic capacitance which would yield increased control, however, this parameter cannot be reduced indefinitely due to the weakening of the piezoelectric patch itself. For these reasons, a thickness of $0.5 \mathrm{~mm}$ was selected. 
Figure 1: Schematic of the plate with its dimensions. Input force applied at point $P_{1}$. Single-point response measurement at $P_{2}$. Areas labeled $\Omega_{1}, \Omega_{2}$, and $\Omega_{3}$ are the upstream, active interface, and downstream sub-domains of the plate.

\subsection{Shunt circuit design}

The results presented in Collet et al. (2012) provide an approach for optimization of the external shunt circuit in terms of the real and the imaginary parts of an imposed synthetic impedance. The authors identified two possible behaviors depending on the criterion used in the optimization. Minimizing the velocity of the energy propagation in the region of the active array it is therefore possible to limit the propagation of the wave beyond the active interface. The optimal circuit is also able to modify the transmission properties of the plate increasing the energy reflected from the active interface. The optimal controlling impedance for this approach requires a negative capacitance with a relatively small negative resistance (Collet et al. (2012)). The negative value of the resistance is due to the control system suppressing the natural damping of the passive structure in order to create a fully reactive periodic system in which a perfect theoretical band gap would exist. As an alternative, choosing a criterion based on exploiting the effect of electrical damping it is possible to modify the dynamic properties of the plate by increasing the energy absorption properties of the active interface. Optimization of the electric synthetic impedance parameters for this criterion (Collet et al. (2012)) leads to design of an absorbing metacomposite able to reduce the vibratory energy flowing through it by increasing the power dissipated within the electric circuit. This optimal case requires a negative capacitance and a positive and larger frequency-dependent resistance as compared to the reactive interface criterion described above (Collet et al. (2012)). However, the required electrical impedances for these two circuit optimization approaches cannot be readily reproduced experimentally because it is not possible to exactly recreate the optimal resistance and capacitance over the whole frequency range of interest.

For the work presented here, the layout of the actual circuit implemented for control of 


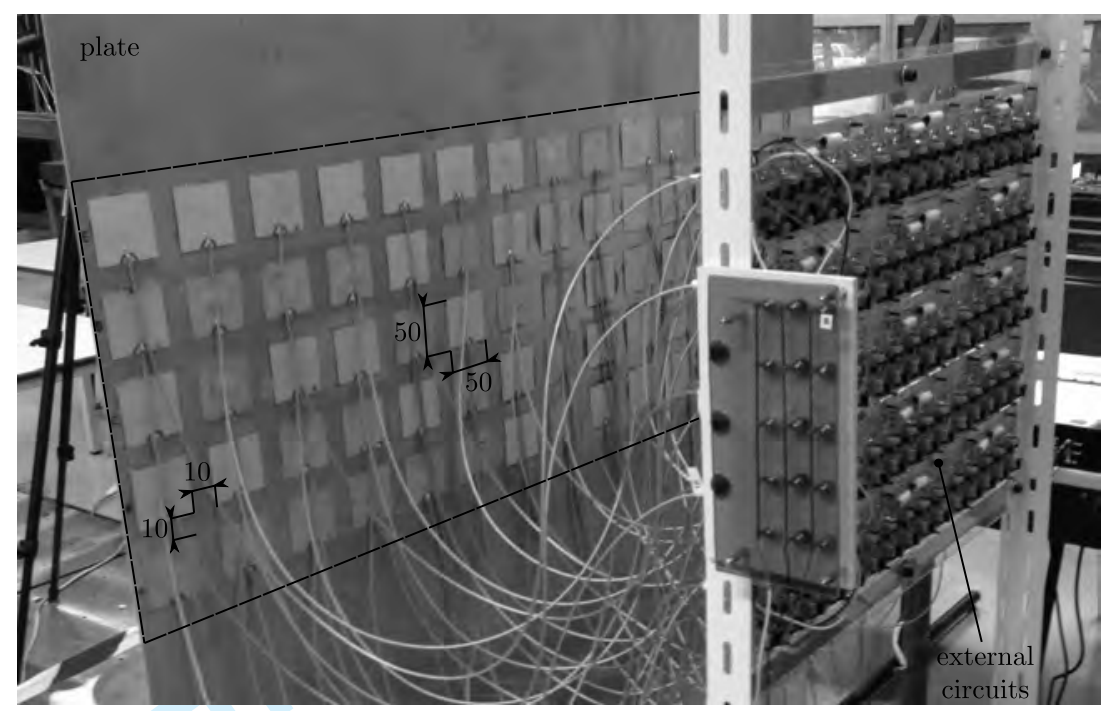

Figure 2: Photograph of plate with 75 element piezoelectric patch array comprising an active interface, connected to bank of 75 individual negative impedance shunt control circuits. Patch dimensions and spacing annotated on photograph.

the patches is presented in Figure 3. This circuit contains a number of passive components, including the resistances $R_{s}, R_{2}, R_{3}, R_{4}$, and the capacitance $C_{2}$, as well as an active component, specifically, an operational amplifier that is essential for the circuit to reproduce the desired negative capacitance behavior. This specific circuit layout was chosen as opposed to others (Fleming et al. (2000)) because of its simplicity and its effectiveness in the frequency range of interest. The impedance of the circuit in Figure 3 is determined by the formula

$$
Z_{e q}(\omega)=R_{s}-\frac{R_{3} R_{2}}{R_{4}\left(1+i \omega R_{2} C_{2}\right)} .
$$

Figure 3 depicts the frequency-dependent behavior of the actual impedance produced by the circuit (Equation 1); in the top of the figure is the equivalent resistance $\left(\operatorname{real}\left(Z_{\text {eq }}\right)\right)$, and at the bottom the equivalent capacitance $\left(1 /\left(-\operatorname{imag}\left(Z_{e q}\right) \omega\right)\right.$ ). Both terms vary as a function of the frequency; in particular the resistance at low frequency presents a negative value that can introduce some instability which can degrade the overall performance of the control system. From the practical point of view, the circuit presented in Figure 3 is further modified by adding some diodes in order to protect the operational amplifier. The resistances $R_{3}$ and $R_{4}$ were implemented through a single potentiometer $R_{\text {pot }}$ with the aim of simplifying the circuit layout. The negative impedance control technique requires tuning of the synthetic capacitance around the capacitance value of the piezoelectric ceramic at free stress condition (Livet et al. (2011)); this value is intrinsic to the electromechanical coupling and depends upon the material properties, the geometry of the patches and the plate stiffness. The intrinsic capacitance of the piezoelectric patch can be estimated by measurement using a capacitance meter when the specimen is free of external forces. In the present case the measured nominal patch capacitance is $52 \mathrm{nF}$. The actual circuit was tuned by varying the position of the potentiometer $R_{p o t}$ in small increments from high negative values of the synthetic capacitance to a value very close to the targeted intrinsic capacitance value. When the external circuit approaches the set-point the system 

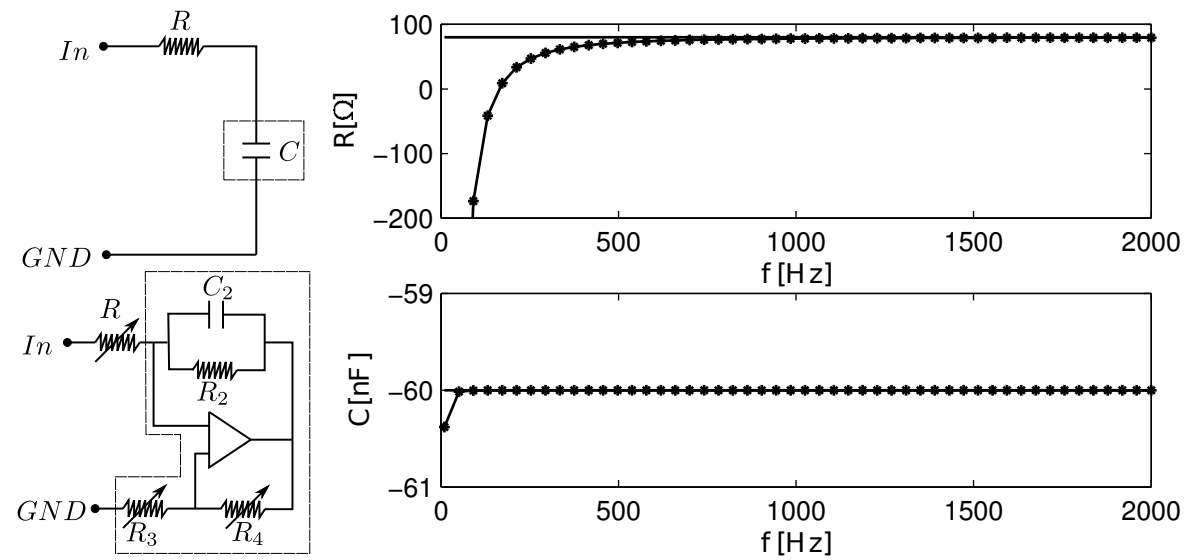

Figure 3: Comparison between the desired performance of the ideal circuit and that of the physically implemented circuit. On the left, basic architecture of circuit. On the right, the circuit's impedance depicted in terms of equivalent resistance (top) and equivalent capacitance (bottom).

tends to be unstable, and an increase of output voltage is observed (Livet et al. (2011)). If the output voltage reaches the limits of the op-amp's power supply voltage then the operational amplifier saturates and its output clips. In order to mitigate this effect, the circuit was modified by adding a complementary circuit able to detect saturation of the output. Basically, a comparator was introduced to monitor the op-amp output in order to identify these voltage overloads (see figure 4 for details). This circuit is based on a comparator that measures the differential input between the positive component of the voltage delivered by the operational amplifier at the output gate and a reference DC signal from the power supply circuit. Whenever the differential signal is greater than zero the comparator's gate is opened, delivering a current able to illuminate a light emitting diode (LED). This simple solution is very helpful since it allows one to detect this particular source of instability, therefore speeding the circuit tuning process. This feature becomes very useful in this context, where a large number of actuators is required. For example, when several piezoelectric patches are excited, a loss of performance can be observed due to the electromechanical coupling occurring between adjacent patches. In this case, observing the state of the LEDs on the control panel it is possible to correctly and efficiently reset each control circuit.

For the work at hand, two configurations of the of circuit have been implemented in order to program two different vibroacoustic behaviors:

- Case 1 corresponding to a lightly-damped, reactive or reflective configuration, is obtained by tuning the negative capacitance $C_{n e g}$ very close to the instability point that corresponds to the opposite of the intrinsic capacitance of the piezoelectric $\left(C_{n e g}=-62 n F\right)$. The resistance $R_{s}$ is set to a small positive value able to reproduce the desired behavior (wave reflection) and still ensuring the stability of the control system $\left(R_{s}=40 \Omega\right)$. In the context of shunted piezoelectrics, this control case implements a "negative stiffness"; in the absence of damping, this yields a more reactive interface than otherwise.

- Case 2: corresponding to an absorbing configuration, and is obtained by tuning the 
negative capacitance $C_{n e g}$ with the same value of Case 1 . The energy absorption enhancement is then obtained by imposing a larger resistance $R_{s}\left(R_{s}=400 \Omega\right)$ at the input to the negative impedance shunt.

In order to choose the more suitable set of electric parameters a preliminary investigation have been done on a plate equipped with a single patch as depicted in Figure 5.

In Figure 6 the mechanical response of the controlled plate is presented for three different configurations: the reference response obtained by considering the circuit OFF (black line), the response of the controlled system assuming the circuit ON Case 1 (red line) and the response of the controlled system assuming the circuit ON Case 2 (blue line). For the control ON Case 1 we observe a shift of the resonance peaks toward lower frequency values this phenomenon is clearly associated to the modification of the stiffness (almost reactive interface), around 300 and $3800 \mathrm{~Hz}$ the resonance peaks are no longer superposed. For the Case 2 the control mechanism is radically different, the mechanical response is attenuated but non stiffness modification is observed (absorbing interface), around 600 and $3200 \mathrm{~Hz}$ the resonance peaks are dramatically reduced. 
Figure 6: Frequency response function of the aluminum plate equipped with a single patch. The curves have been obtained by varying the resistance $R_{s}$ for three different configurations: control OFF (black line), control ON Case 1 (red line) and control ON Case 2 (blue line).

\section{Experimental set-up}

As noted previously, the structure of interest, shown in Figure 2, consists of an aluminum plate equipped with 75 piezoelectric actuators connected to external circuits tuned with the identical set of circuital parameters and arranged so as to form a periodic array of $5 \times 15$ units. Standard epoxy glue was used to bond the piezoelectric ceramics to the top surface of the plate as described in Figure 4.

Elastic waves are excited by a shaker located in the lower corner of the plate, $680 \mathrm{~mm}$ away from the active interface at the point labelled $P_{1}$ in Figure 7 . The input transducer is driven by an input signal consisting of a random broad-band excitation and amplified by an LDS power amplifier. The response of the structure to the input disturbance and action of the active interface was measured with a scanning laser vibrometer (Polytec PSV-400). Measurements were conducted over a grid of $37 \times 81$ scan points with a spatial resolution of $20 \mathrm{~mm}$ in the horizontal and vertical directions. At each grid point, the laser measured the time history of the out-of-plane velocity $\dot{w}(x, y, t)$.

The measured velocity signal was processed using a Fourier transform; the obtained signal $\dot{W}(x, y, \omega)$ depends on the coordinates $x, y$ and the angular frequency $\omega$. By means of an impedance head the input excitation force was also measured. The Fourier transformed signals $F(\omega)$ and $\Gamma(\omega)$, representing the excitation force and the acceleration at the force input point labeled as $P_{1}$ on Figure 7 , were used for estimation of the power injected into the system. Another quantity of interest obtained during the post-processing is the mobility function given by

$$
Y(x, y, \omega)=\frac{\dot{W}(x, y, \omega)}{F(\omega)} .
$$



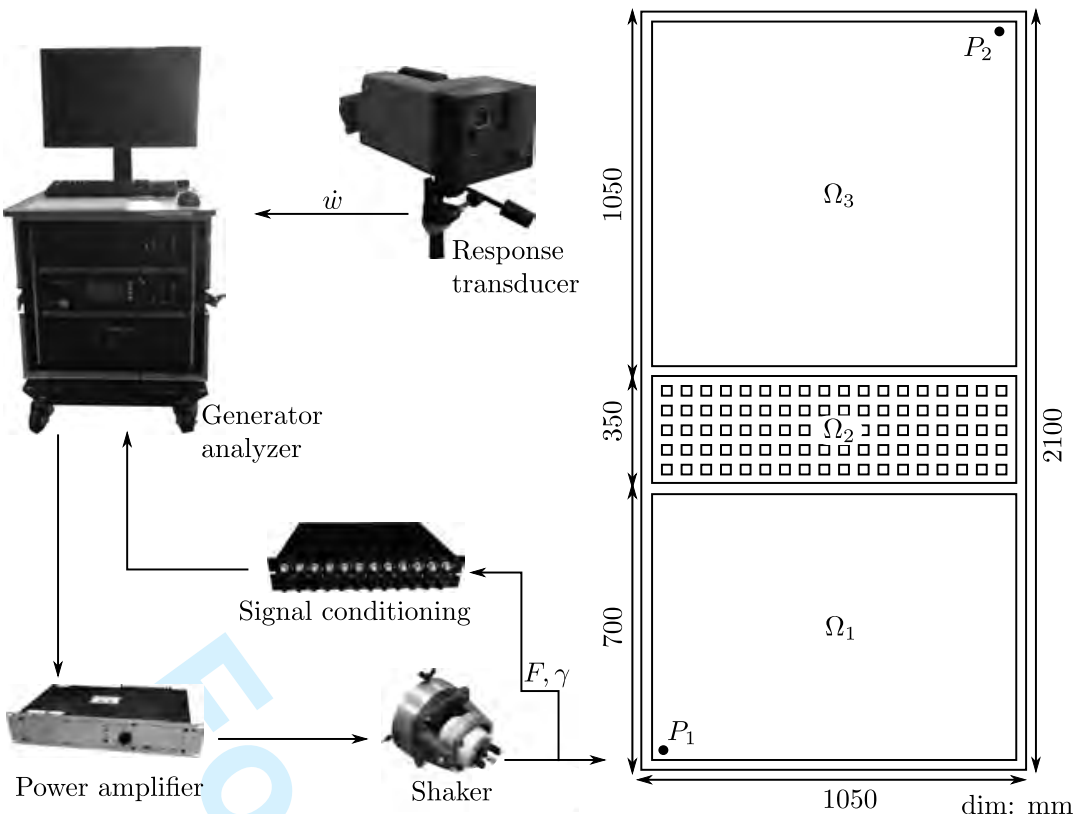

Figure 7: Layout of FRF measurement system. $P_{1}$ is the force input position, while $P_{2}$ used for a representative single-point transfer mobility measurement.

\section{Results and discussions}

Selected experimental results are presented here in order to demonstrate the effectiveness of the proposed active interface and distributed control technique. First, the results obtained performing a single-point mobility measurement will be presented for introductory purposes. A full-field measurement will further illustrate the main features of the control system, and finally some consideration of the energetics of the approach will conclude the section, characterizing the performance of the considered metacomposite.

\subsection{Single-point mobility measurement}

Mobility measurements permits a ready assessment of some specific features of the system. Figure 8 depicts the plate's transfer mobility frequency response function, measured at point $P_{2}$ of the controlled plate (Figure 7 ). The mobility function is measured over the frequency range of $0-5000 \mathrm{~Hz}$ along with its coherence. In the low frequency range, $0-500 \mathrm{~Hz}$, the system shows a degradation of coherence; therefore, the value of the mobility function is less reliable in this frequency region. For this reason, in the following section, the analysis of the system for higher frequency values will be emphasized, focusing in particular on the $500-5000 \mathrm{~Hz}$ frequency range. Moreover, the system's response function clearly shows a high modal density corresponding to mid-frequency behavior that makes classical control methods difficult to apply.

\subsection{Third octave mobility comparison}

Due to the high modal density, comparing the different response functions while keeping the same frequency resolution will be quite problematic due to the action of the control 
restructuring the modal behavior of the plate. For this reason, a different approach has been employed here for presentation of the results. Each frequency response function or metric of interest (e.g., loss factor) has been expressed in terms of band-averaged third octave bands to lessen the impact of modal restructuring on the presentation of results, while still permitting assessment of important system features (an analysis of the modal density of the plate indicated that there were at least three modes per third octave band starting with the $40 \mathrm{~Hz}$ band).

Figure 9 depicts the difference in the third-octave band averaged mobility magnitude in $\mathrm{dB}$ between the reference response (control OFF) to the controlled response for the two cases presented in the previous section (Cases 1 and 2). Positive values correspond to an attenuation of the system's response. The figure presents two plots: the upper plot is the difference in input mobility at the excitation point $P_{1}$, while the lower plot is the difference in the transfer mobility between the input point $P_{1}$ and a response point $P_{2}$ (Figure 7 ). The response point $P_{2}$ considered here is at the opposite corner of the plate from the input, and located downstream with respect to the active interface. Considering the input mobility, the impact of the control for both cases is relatively minor, on the order of $\pm 1 \mathrm{~dB}$ in all bands. But, considering the response at $P_{2}$ on the downstream side of the active interface, we observe an enhancement of control capabilities in specific frequency ranges. For example in Case 1 the performances of the control system, in terms of vibration suppression, is almost always significantly lower compared to the Case 2 except around $2000 \mathrm{~Hz}$. For Case 2 better performance is obtained, above about $100 \mathrm{~Hz}$ the system systematically adds at least $5 \mathrm{~dB}$ reduction. Some bands display significantly better results; for example, a $15 \mathrm{~dB}$ reduction around $2000 \mathrm{~Hz}$ for Case 1, the most reactive control configuration. The control for Case 2, the absorptive control configuration, does not achieve as great a maximum single-band control as does Case 1 at $2000 \mathrm{~Hz}$, but it exhibits a much broader frequency range of control. It is clear that in the frequency band $50-5000 \mathrm{~Hz}$ the control system 
works effectively, reducing the magnitude of the vibrations. The frequency band where the control system actually reduces the vibrations depends on the circuit's configuration; small values of the input shunt resistance $R_{s}$ (Case 1) impact higher frequency values in a narrow band. Larger values of the input shunt resistance $R_{s}$ (Case 2) impact a broader frequency range.

These results illustrate an inherent difference between the control configurations between the two cases; the more reactive the boundary, the greater the possible control but in a relatively narrow frequency band; the more absorptive the boundary, the greater the bandwidth of control with some reduction of maximum achievable control. Further, these results show the effectiveness of the proposed control strategies, and illustrates a basis for selection between them for a given application; if one seeks to control a narrow-band disturbance, then the reactive control configuration would be appropriate. If the disturbance is broad-band, then the absorptive control would be appropriate.

\subsection{Full-field mobility measurement}

The results presented in the previous section cannot fully demonstrate the impact or effectiveness of the control system because they are limited to selected single point measurements. Figure 10 presents measurements of the plate's mobility as a function of the spatial coordinates $x$ and $y$ at $25 \mathrm{~Hz}$. The top row of images presents, left to right, the mobility of the plate for the reference measurement (control OFF), the field with the Case 1 control and the field for the Case 2 control, all at $25 \mathrm{~Hz}$. The control system acts differently 

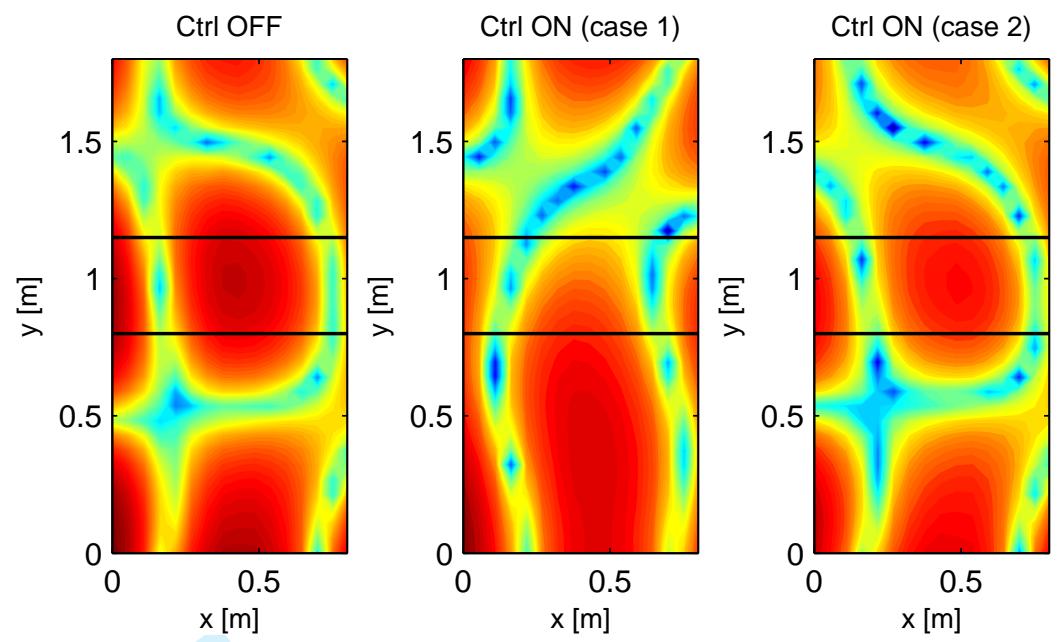

Figure 10: Control OFF vs. control ON full-field mobility measurement. (Top) response at $25 \mathrm{~Hz}$; (bottom) response at $1680 \mathrm{~Hz}$. Horizontal black lines indicate location of active interface.

according to the circuit's setting. For the Case 2 control configuration the behavior of the plate is not affected by the control system, and the plate's operational deflection shape is unmodified, with only an incremental reduction of the vibration amplitude observed. In contrast, for Case 1, the dynamic response of the plate is modified, and a different response shape is observed due to modal restructuring.

\subsection{Loss factor estimation}

It is informative to consider the loss factor exhibited in the plate response as a function of the control configuration. Estimation of the loss factor requires a measure of the timeaveraged power input to the system, and the total time-averaged energy of the system (potential plus kinetic energy). The experimental set-up allows the measurement of the input force and the response velocity at the driven point, along with the velocity field over the entire plate. Using this information, it is possible to calculate the direct component (time-averaged) of the power flow into the system (Bobrovnitskii (1999)):

$$
P_{0}=\operatorname{real}\left(\frac{1}{2} F \cdot v^{*}\right) .
$$

Further, the integration of the velocity field over the entire surface of the plate yields an estimate of the plate's time-averaged kinetic energy,

$$
T_{0}=\int_{\Omega} \rho v v^{*} d V,
$$

and the time-averaged total energy $E_{0}$,

$$
E_{0}=T_{0}+U_{0} \simeq 2 T_{0} .
$$

$E_{0}$ is estimated by using SEA hypotheses that remain valid only at the resonance or for higher frequencies where the modal density is sufficiently high (Bobrovnitskii (1999)). The 


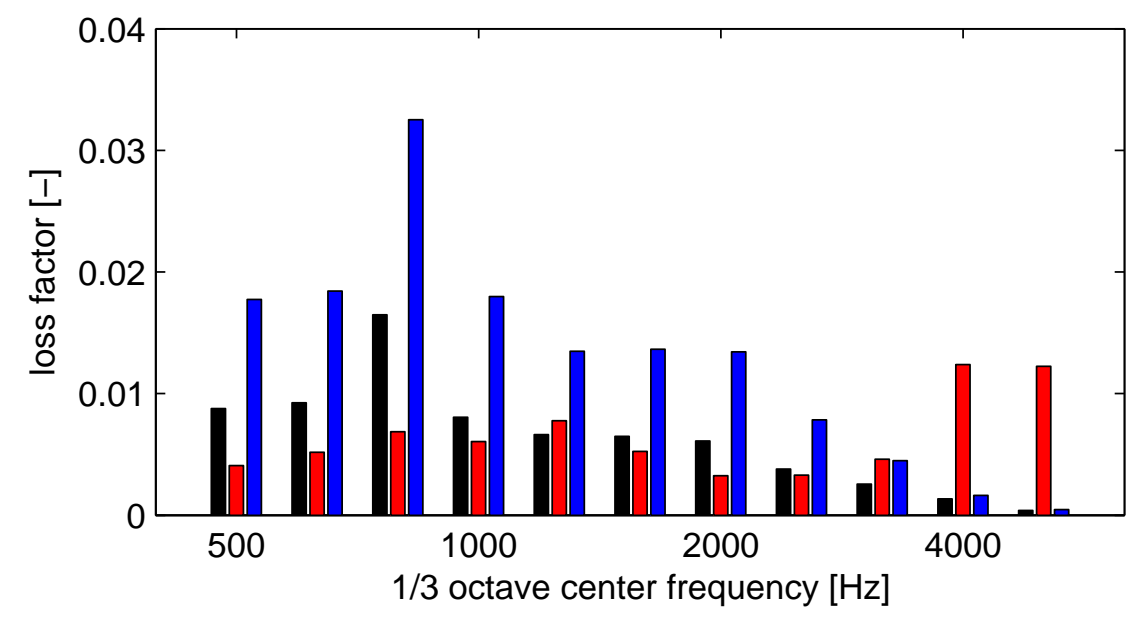

Figure 11: 1/3 octave band averaged loss factor of the structure: (black) control OFF; (red) control ON Case 1; (blue) control ON Case 2.

loss factor of the plate is then calculated by

$$
\eta=\frac{P_{0}}{\omega E_{0}},
$$

where $P_{0}$ also represents the time-averaged power dissipated in the system.

Figure 11 summarizes the estimated loss factor for the three different control conditions considered here: control OFF, Case 1 and Case 2. As with the mobility measurements presented earlier, the loss factor data is presented here in terms of third octave band averaged values. For the control OFF case, the loss factor is no more than 0.015 below $1000 \mathrm{~Hz}$, and falls to lower values at high frequencies. For the reactive, low-damping configuration of Case 1, the system is less damped than the uncontrolled configuration in all bands below $4000 \mathrm{~Hz}$. For the $4000 \mathrm{~Hz}$ band and above, the damping increases from significantly less than 0.01 to slightly greater than 0.01 . This behavior is a consequence of the frequency-dependent reactive nature of the control suppressing the plate's loss factor at lower frequencies, while the still non-zero dissipation of the control adds damping at the higher frequencies. For the higher resistance $R_{s}$ configuration of Case 2 , the system is more strongly damped over the whole frequency band of interest. However, the best results are obtained over the bands from $500 \mathrm{~Hz}$ to $2500 \mathrm{~Hz}$, where the loss factor with the control ON exceeds that with the control OFF. Similar results where obtained by Beck et al. (2011) on a beam structure.

To further illustrate the impact of the control ON the system, consider Figure 12 which presents the difference, $\Delta \eta$, between the loss factors for each of the controlled systems (Case 1 and 2) and the loss factor for the corresponding third-octave band for the control OFF condition. A positive value of $\Delta \eta$ means that the control system increased the loss factor for the band, while negative value means that the control system has reduced the loss factor for the band. This presentation clearly brings out that the reactive configuration of Case 1 reduces the loss factor for frequencies below $2000 \mathrm{~Hz}$ and increases it for the $4000 \mathrm{~Hz}$ and higher bands. The absorptive configuration of Case 2 does decrease the loss factor below $125 \mathrm{~Hz}$, but increases the loss factor from $200 \mathrm{~Hz}$ through the $3500 \mathrm{~Hz}$ band, reflecting the broader bandwidth of control of Case 2 . 


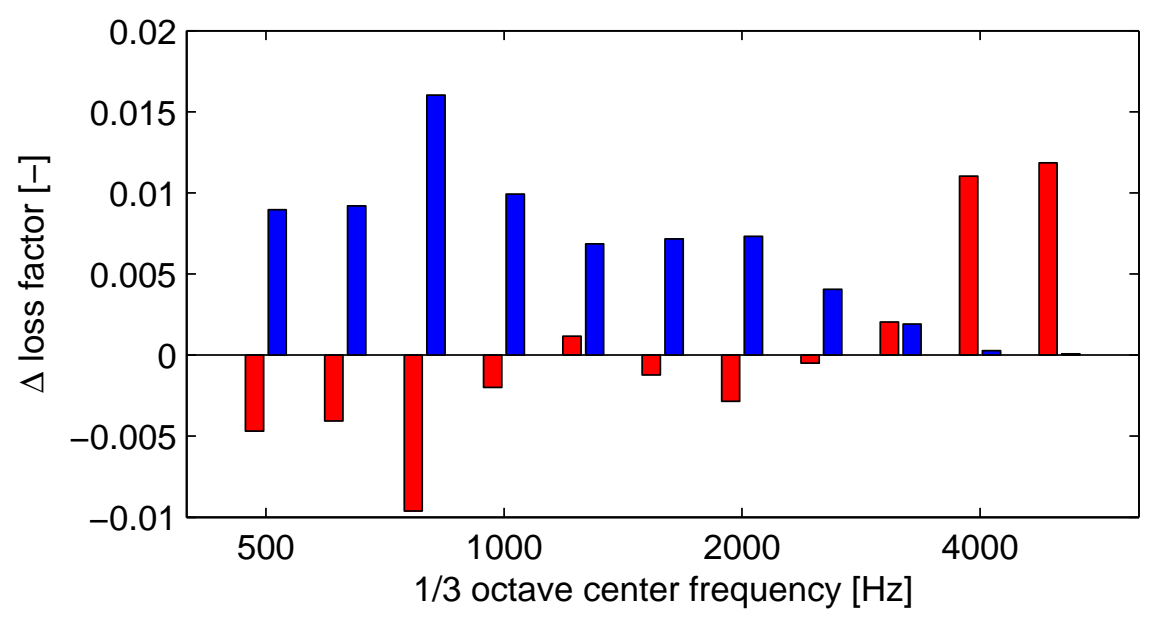

Figure 12: Difference in 1/3 octave band averaged loss factor between the control ON and control OFF cases: (red) control ON Case 1 vs. control OFF; (blue) control ON Case 2 vs. control OFF.

\subsection{Kinetic energy distribution}

The loss factor evaluation presented in the previous section can be used to evaluate the fraction of energy dissipated in each cycle of vibration, but no information about the modification of the plate's internal dynamics within each of the regions of the plate (upstream of the active interface, within the active interface, and downstream of the active interface) can be obtained. In order to address this aspect of the influence of the control scheme, if one computes the time-averaged kinetic energy over a subdomain of the plate $\Omega_{i}$, it is possible to evaluate the kinetic energy distribution within the system by calculating the ratio

$$
\tau_{i}=\frac{T_{0}^{\Omega_{i}}}{T_{0}^{\Omega_{t o t}}}
$$

where $\Omega_{t o t}$ represents the plate's total area and $\Omega_{i}$ the different sub-domains of interest on the plate; below the active interface (containing the force disturbance input point), within the active, interface, and above the active interface. These subdomains are labeled on Figure 7. Note that in proportion to the total area of the plate, each subdomain's fraction of the plate area are $0.33,0.17,0.50$, for the upstream, interface, and downstream areas, respectively.

As for the loss factor considered earlier, since what is of interest here is the impact of the control on the system, Figure 13 depicts the difference in the kinetic energy ratio $\tau$ calculated for each of the subdomains of interest, and for each control circuit configuration with respect to the control OFF configuration. Negative values for the difference indicate that the kinetic energy has decreased in the subdomain relative to the control OFF configuration, while positive values indicates it has increased. While not evident from Figure 13, with the control OFF the kinetic energy ratio was roughly uniform with frequency and proportional to the area of each subdomain, as would be expected for a diffuse band-averaged wavefield. However, with the control ON the vibratory energy of the plate is distributed differently within the plate as compared to the control OFF condition, and differently for each control configuration. 
Considering each subdomain in turn, starting with the downstream area of the plate, represented by the top plot of Figure 13, with the Case 1 control there is little impact on the kinetic energy distribution until above $3000 \mathrm{~Hz}$, where the increasingly negative values of the difference indicates that less of the plate's total kinetic energy is in the downstream subdomain. However, for the higher-damped Case 2 control, the negative values of difference in the kinetic energy distribution ratio within the downstream subdomain is reduced over a much broader frequency span from below $500 \mathrm{~Hz}$ to above $3000 \mathrm{~Hz}$. An implication of the reduction of the kinetic energy distribution ratio, relative to the total for these two control cases, is that the kinetic energy distribution ratio had to increase elsewhere on the plate, that is, the energy was localized elsewhere.

Considering the subdomain comprising the active interface, addressed in the middle plot of Figure 13, the kinetic energy distribution within this subdomain as reflected by the plotted difference in the ratio is relatively unaffected to the state and configuration of the control.

Finally, considering the upstream subdomain containing the input force, addressed in the bottom plot of Figure 13, the behavior is effectively opposite of what is observed in the downstream subdomain, as it must in consideration of conservation of energy. For both control configuration cases, the fraction of the total kinetic energy of the plate is greater in this upstream subdomain, clearly indicating that the active interface is serving to isolate the downstream section from the energy in the upstream section. The degree of that isolation depends on the form of the control, with the absorbing configuration, Case 2, yielding a greater degree of isolation over most of the frequency range, up to approximately $3000 \mathrm{~Hz}$. Above $3000 \mathrm{~Hz}$ the Case 1 configuration yields better isolation as compared to Case 2; note that this frequency dependence is also reflected in the loss factors for these two cases, as discussed in the previous section.

A clear depiction of the impact of the active interface may be obtained by considering the area distribution of kinetic energy (essentially plotting the square of the local velocity on the plate) as depicted in Figure 14. The top row of plots depict the kinetic energy distribution among the three control configurations at $2500 \mathrm{~Hz}$. On the left, (control $\mathrm{OFF}$ ) the kinetic energy is evenly spread over the entire plate. In the middle, the system's response is slightly modified by the low-damping, low resistance $R_{s}$ control Case 1 . In contrast, as a result of increasing the value of the resistance $R_{s}$ for Case 2 , there by increasing the damping, the kinetic energy distribution of the plate is clearly modified; the response is most strongly confined to the portion of the plate below the active interface. Considering a higher frequency example, for instance $3000 \mathrm{~Hz}$ as depicted in the lower plots of Figure 14, the behavior of the system is radically modified. For the lightly-damped control Case 1, the vibratory energy of the plate is even more noticeably confined in the upstream subdomain $\Omega_{1}$ as compared to the response at $2500 \mathrm{~Hz}$. In fact, the vibration amplitude is increased in the upstream region for Case 1 as compared to the control OFF case. For the more heavily-damped control of Case 2, the reflective behavior is no longer present, and the driving control mechanism is a dominantly dissipative effect that makes the kinetic energy field weaker everywhere through suppression of a reactive vibratory response.

\subsection{Power injected}

Figure 15 depicts the frequency behavior of difference in the real injected power flow into the system in third octaves, while Figure 16 depicts the difference in the reactive power. 
As previously, the depiction is in terms of the difference between the control ON cases and the control OFF case. As compared to the power injected with the control OFF, the power injection behavior is significantly different for the two control cases. For the reactive condition, Case 1, the real and reactive input power actually increases, indicating a change in the input impedance presented to the forcing. For the damped Case 2, there is almost no change in the real and reactive input power, indicating effectively no change to the input impedance.

\section{Conclusions}

The control capability of an active interface between two regions of a plate with either a lightly-damped or more heavily damped configuration has been demonstrated through experimental evidence.

The active interface and control technique strongly modifies the energy distribution properties of the structure. The introduction of the active interface allows one to enhance the absorbing or reflecting properties of the plate considered as a waveguide, particularly for higher frequencies. These properties strongly depend on the configuration of the control circuit, between lightly damped and more heavily damped. Through analysis of these two control configurations (lightly damped Case 1, and more heavily damped Case 2), the following conclusions can be drawn:

- the absorption properties of this metacomposite are enhanced in both cases.

- the transmission of the power flow at the active interface can be controlled by manipulating the external circuit, i.e. modification of reflective properties versus added damping

- the frequency band where the system is effective can be shifted by modifying specific values of the external circuit, i.e. $1000-3000 \mathrm{~Hz}$ (Case 2) $3000-5000 \mathrm{~Hz}$ (Case 1)

- it is possible to alter the elastic properties of the metacomposite (modal restructuring)

Therefore, this control technique could be used effectively in applications where the isolation of a source of disturbance is of paramount importance. This work demonstrates that, under specific conditions, it is indeed possible on extended structures to isolate a disturbance response to a limited region of the overall structure, and to do so without significantly modifying the impedance "perceived" by the excitation source.

An implication of the results presented here on future research is that the reflective properties of the active interface could also be used in an energy harvesting context; a confinement of the kinetic energy at the position of an energy harvester would allow it to recover more energy in each cycle as compared to without such confinement.

Also with respect to future studies, it will be interesting to estimate the quantity of vibrational energy dissipated within the electric circuit and to characterize the control system from the perspective of wave propagation through measurement of wave dispersion and scattering.

\section{Acknowledgments}

The authors would like to thank the French National Agency for granting the CALIOP project number NT09-617542 and the Labex Action. 


\section{References}

Ambati, M., N. Fang, C. Sun, and X. Zhang (2007). Surface resonant states and superlensing in acoustic metamaterials. Physical Review B 75(19), 195447.

Beck, B., K. Cunefare, M. Ruzzene, and M. Collet (2011). Experimental analysis of a cantilever beam with a shunted piezoelectric periodic array. Journal of Intelligent Material Systems and Structures 22(11), 1177-1187.

Bobrovnitskii, Y. (1999). Some energy relations for mechanical systems. In F. Fahy and W. Price (Eds.), IUTAM Symposium on Statistical Energy Analysis, Number 67 in Solid Mechanics and Its Applications, pp. 37-46. Springer Netherlands.

Casadei, F., M. Ruzzene, L. Dozio, and K. Cunefare (2010). Broadband vibration control through periodic arrays of resonant shunts: experimental investigation on plates. Smart Materials and Structures 19(1), 150.

Chen, S., G. Wang, J. Wen, and X. Wen (2013). Wave propagation and attenuation in plates with periodic arrays of shunted piezo-patches. Journal of Sound and Vibration 332(6), 1520-1532.

Collet, M., M. Ouisse, and M. Ichchou (2012). Structural energy flow optimization through adaptive shunted piezoelectric metacomposites. Journal of Intelligent Material Systems and Structures 23(15), 1661-1677.

Collet, M., M. Ouisse, M. N. Ichchou, and R. Ohayon (2012). Semi-active optimization of 2D wave dispersion into shunted piezo-composite systems for controlling acoustic interaction. Smart Materials and Structures $21(9), 94$.

Fang, N., D. Xi, J. Xu, M. Ambati, W. Srituravanich, C. Sun, and X. Zhang (2006). Ultrasonic metamaterials with negative modulus. Nature Materials 5(6), 452-456.

Fleming, A., S. Behrens, and S. Moheimani (2000). Synthetic impedance for implementation of piezoelectric shunt-damping circuits. Electronics Letters 36(18), 1525-1526.

Forward, R. (1979). Electronic damping of vibrations in optical structures. Applied Optics 18(5), 690-697.

Fukada, E., M. Date, K. Kimura, T. Okubo, H. Kodama, P. Mokry, and K. Yamamoto (2004). Sound isolation by piezoelectric polymer films connected to negative capacitance circuits. IEEE Transactions on Dielectrics and Electrical Insulation 11(2), 328-333.

Hagood, N. and A. von Flotow (1991). Damping of structural vibrations with piezoelectric materials and passive electrical networks. Journal of Sound and Vibration 146(2), 243268.

Hu, X., C. Chan, and J. Zi (2005). Two-dimensional sonic crystals with helmholtz resonators. Physical Review E 71(5), 556.

Kim, J. and Y. Jung (2006). Broadband noise reduction of piezoelectric smart panel featuring negative-capacitive-converter shunt circuit. The Journal of the Acoustical Society of America 120(4), 2017-2025. 
Liu, Z., X. Zhang, Y. Mao, Y. Zhu, Z. Yang, C. Chan, and P. Sheng (2000). Locally resonant sonic materials. Science 289(5485), 1734-1736.

Livet, S., M. Collet, M. Berthillier, P. Jean, and J. Cote (2011). Structural multi-modal damping by optimizing shunted piezoelectric transducers. European Journal of Computational Mechanics 20(1-4), 73-102.

Park, C. and A. Baz (2005). Vibration control of beams with negative capacitive shunting of interdigital electrode piezoceramics. Journal of Vibration and Control 11(3), 331-346.

Spadoni, A., M. Ruzzene, and K. Cunefare (2009). Vibration and wave propagation control of plates with periodic arrays of shunted piezoelectric patches. Journal of Intelligent Material Systems and Structures 20(8), 979-990.

Thorp, O., M. Ruzzene, and A. Baz (2005). Attenuation of wave propagation in fluid-loaded shells with periodic shunted piezoelectric rings. Smart Materials and Structures 14(4), 594.

Toffoli, T. and N. Margolus (1991). Programmable matter: Concepts and realization. Physica D: Nonlinear Phenomena 47(1-2), 263-272.

Yang, S., J. Page, Z. Liu, M. Cowan, C. Chan, and P. Sheng (2002). Ultrasound tunneling through 3D phononic crystals. Physical Review Letters 88(10), 104301. 

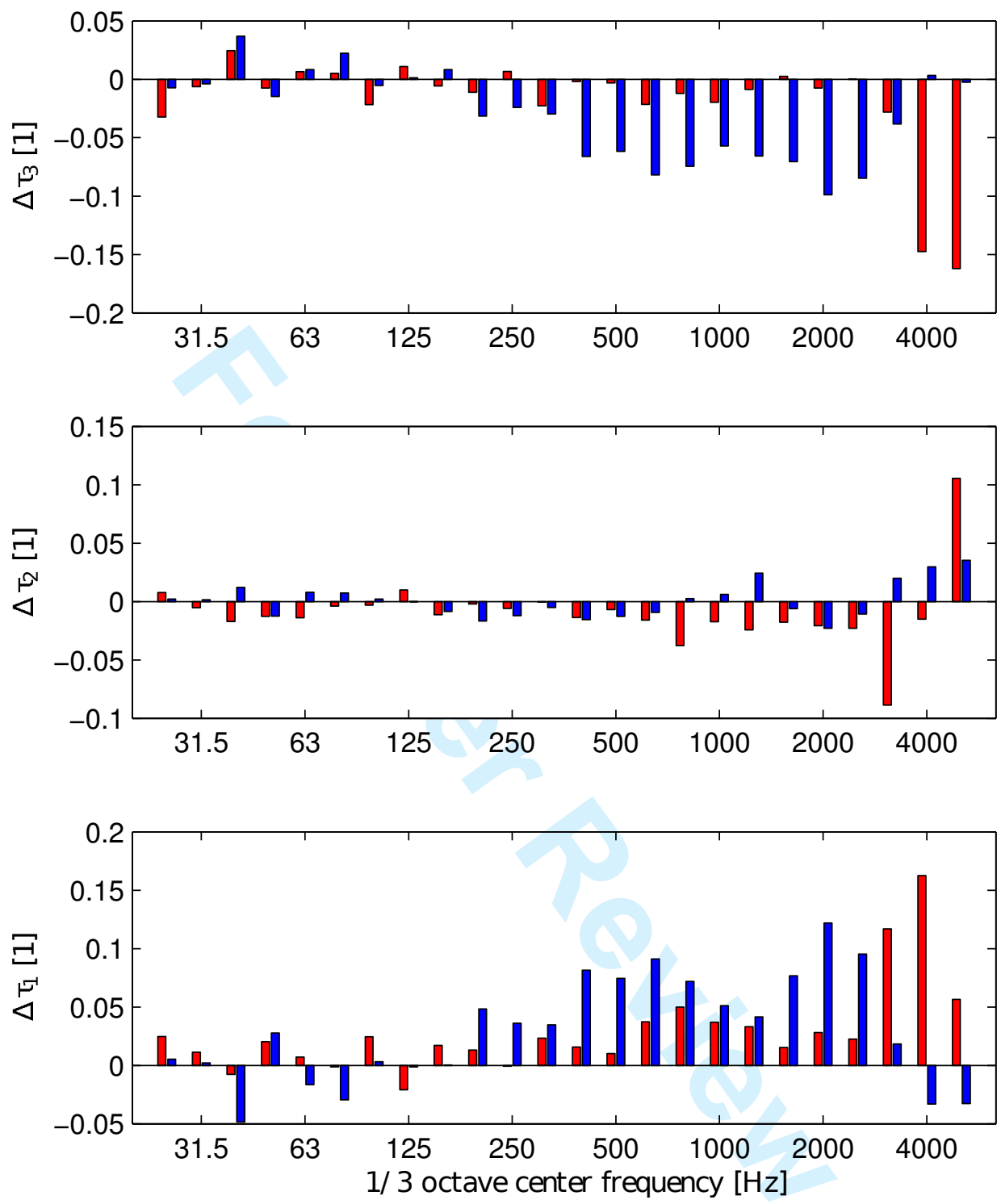

Figure 13: Difference in $1 / 3$ octave band ratio of averaged kinetic energy distribution in each subdomain to the total kinetic energy: (red) control ON Case 1; (blue) control ON Case 2. (Top) subdomain 3, downstream of interface; (middle) subdomain 2, active interface; (bottom) subdomain 1, upstream of interface 

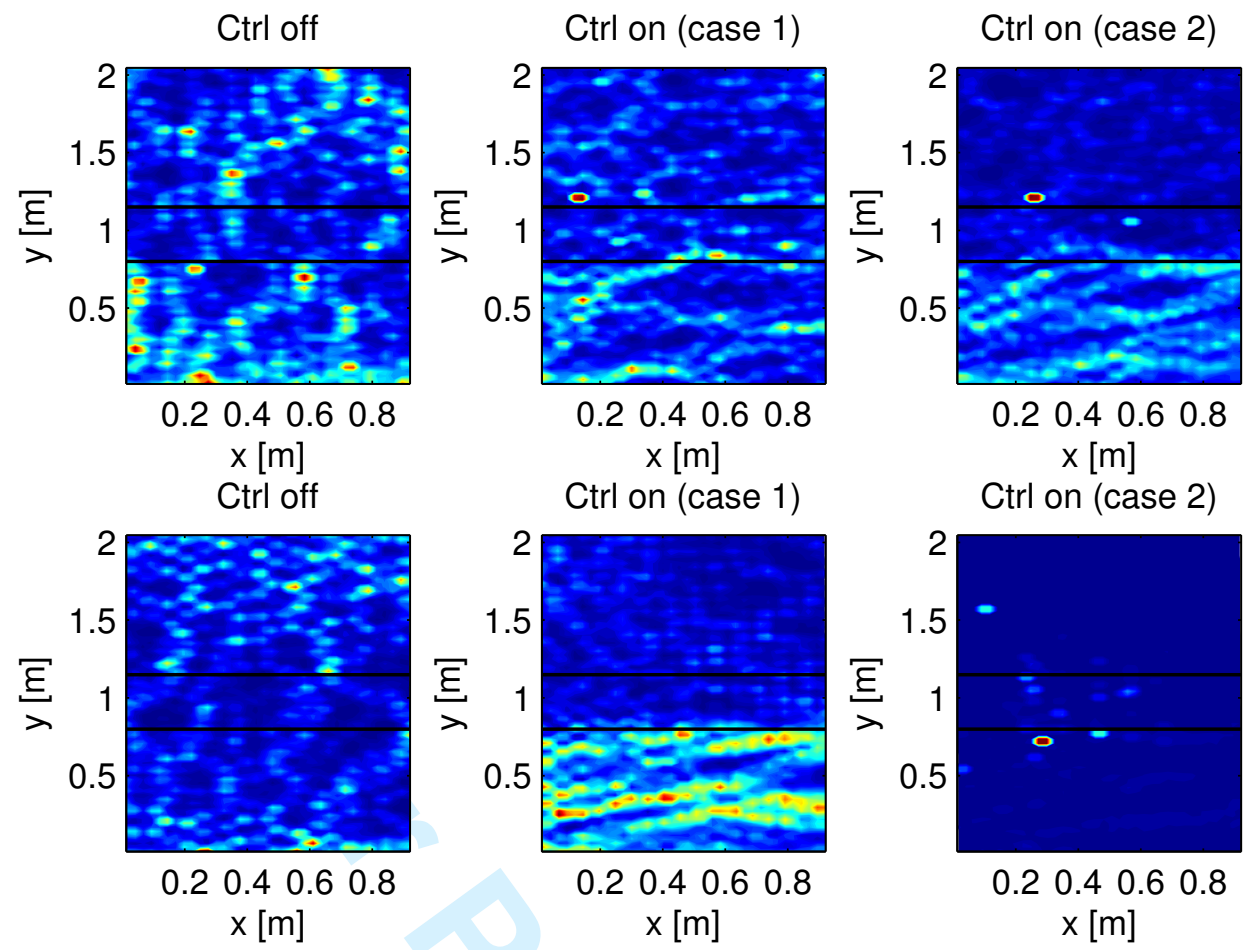

Figure 14: Plate kinetic energy field: (left) control OFF, (center) control ON Case 1, (right) control ON Case 2. (Top) $2500 \mathrm{~Hz}$, (bottom) $3000 \mathrm{~Hz}$. Horizontal black lines indicate location of active interface.

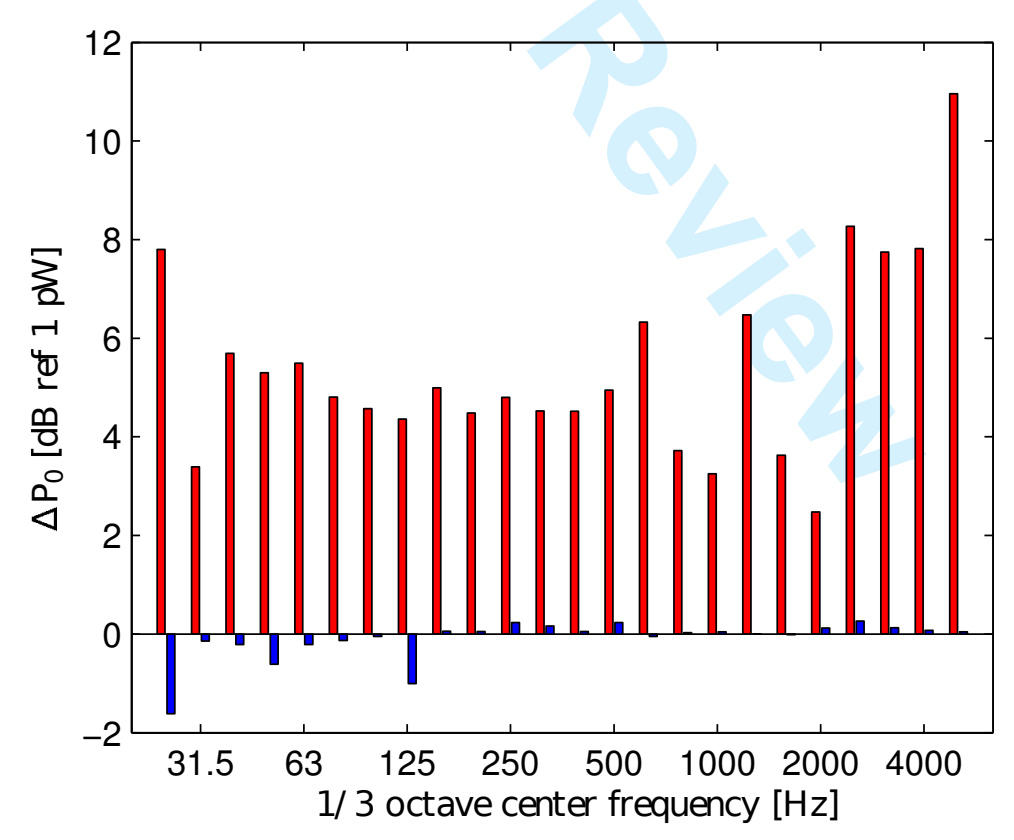

Figure 15: 1/3 octave band averaged and time averaged real power flow into the plate: (red) control ON Case 1; (blue) control ON: Case 2. 


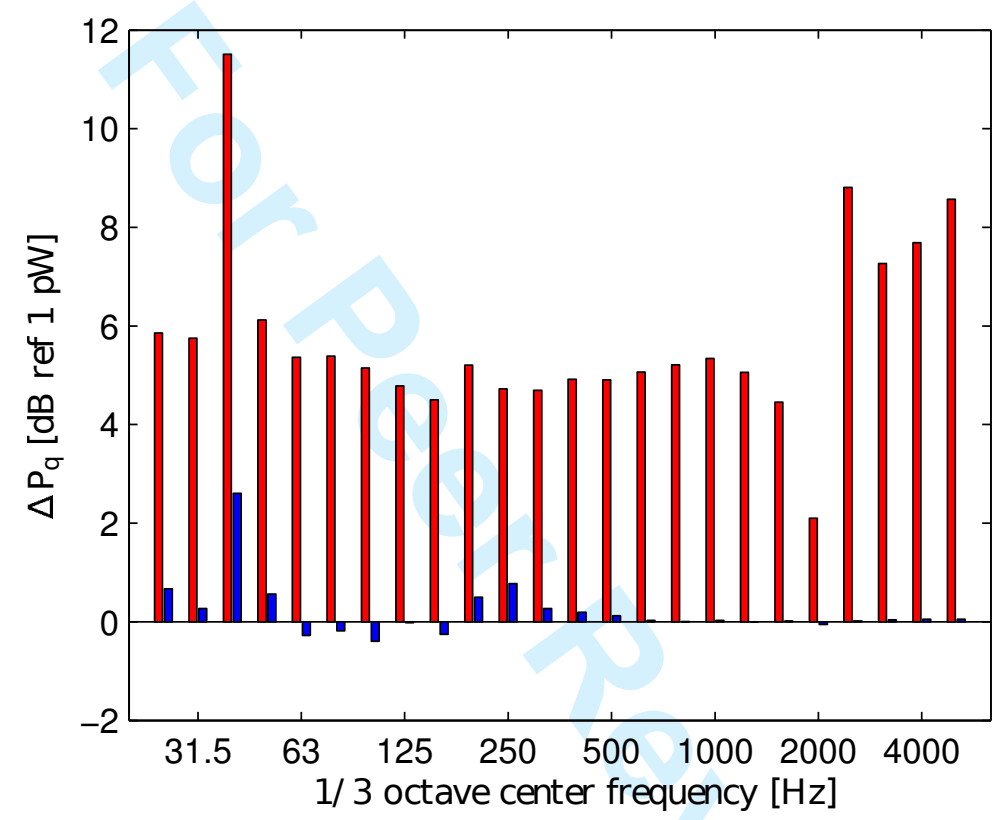

Figure 16: Difference in 1/3 octave band averaged time peak reactive power flow into the plate: (red) control ON Case 1; (blue) control ON Case 2. 